\title{
CONTINUIDADES Y DISCONTINUIDADES EN EL TRABAJO DEL ACOMPAÑANTE TERAPÉUTICO EN CONTEXTOS DE LUCHA DOCENTE Y DE PANDEMIA DE COVID-19 EN ESCUELAS PÚBLICAS DE LA PROVINCIA DE CHUBUT
}

\author{
Gladis Noemí Díaz * \\ Universidad Nacional de la Patagonia San Juan Bosco, Argentina \\ diazgladisn@gmail.com \\ Cristina Del Valle Pereyra ** \\ CONICET, Argentina \\ pereyra.cristina@gmail.com \\ Cynthia Soledad Neun *** \\ Profesorado de Nivel Inicial y de Educación Especial con Orientación en Ciegos y Disminuidos \\ Visuales ISFD N802, Argentina \\ cynthiasneun@gmail.com
}

Recibido: 9/06/2020 - Aceptado: 6/11/2020

\section{Resumen}

En este artículo presentamos un avance en el análisis de las modalidades que asume el trabajo del acompañante terapéutico que interviene con niños y niñas con discapacidad en escuelas públicas, en contextos de "lucha docente" y de pandemia de COVID-19 en la ciudad de Comodoro Rivadavia, provincia de Chubut. Construimos estas ideas en el marco del Proyecto de Investigación 'Los 'apoyos' a alumnos/as. Dispositivos alternativos para la inclusión educativa en las escuelas de educación común". En este estudio nos proponemos conocer las tramas socioeducativas que se configuran en las prácticas que despliegan estos "apoyos" en la cotidianeidad escolar. Desde un enfoque de investigación socio antropológico, desarrollamos el trabajo de campo en escuelas primarias y realizamos la construcción de la información a partir de entrevistas, observaciones y registros de campo.

El recorte del estudio que aquí desarrollamos nos permite conocer las continuidades y discontinuidades en las que se reconfiguran las tareas del acompañante terapéutico, en un contexto de crisis estructural del sistema educativo de Chubut y en el que se imbrican las complejas prácticas educativas en tiempos de pandemia de COVID-19.

Palabras clave: Acompañante terapéutico - Educación - Lucha Docente - Pandemia COVID-19Inclusión.

\footnotetext{
* Docente Investigadora Regular de la Facultad de Humanidades y Ciencias Sociales. Universidad Nacional de la Patagonia San Juan Bosco. Cátedras Psicopedagogía y Práctica Profesional Il de la Carrera de Ciencias de la Educación. Profesora y Licenciada en Ciencias de la Educación. Facultad de Humanidades y Ciencias Sociales. Universidad Nacional de la Patagonia San Juan Bosco Licenciada en Psicopedagogía. Facultad de Filosofía Ciencias de la Educación y Humanidades. Universidad de Morón.

${ }^{* *}$ Doctora en Ciencias de la Educación UBA. Profesora y Licenciada en Ciencias de la Educación Nacional de la Patagonia San Juan Bosco. Becaria Post Doctoral de CONICET en el Centro de Investigaciones y transferencia Golfo San Jorge. Investigadora en el Instituto de Estudios Sociales y Políticos de la Patagonia.

*** Docente en el Profesorado de Nivel Inicial y de Educación Especial con Orientación en Ciegos y Disminuidos Visuales ISFD N`802. Cátedras: Práctica Profesional IV. Profesora y Licenciada en Ciencias de la Educación UNPSJB. Diploma Superior en Curriculum y prácticas escolares en contexto FLACSO. Doctoranda en Doctorado en Ciencias Sociales y Humanas. Facultad de Humanidades y Ciencias Sociales. Universidad Nacional de la Patagonia San Juan Bosco. Investigadora en la Unidad ejecutora de Proyectos de Investigación de la Secretaría de Ciencia y Técnica Nacional de la Patagonia San Juan Bosco, vinculados a los procesos de inclusión educativa y las relaciones entre educación común y especial.
} 


\title{
CONTINUITIES AND DISCONTINUITIES IN THE WORK OF THE THERAPEUTIC COMPANION IN CONTEXTS OF TEACHER STRUGGLE AND COVID-19 PANDEMIC IN PUBLIC SCHOOLS OF THE PROVINCE OF CHUBUT
}

\begin{abstract}
In this article we present an advance in the analysis of the modalities assumed by the work of the therapeutic companions who intervene with children with disabilities in public schools in contexts of "teacher struggle" and of the COVID-19 pandemic in the city of Comodoro Rivadavia, province of Chubut. We build these ideas within the framework of the Research Project "Supports to students. Alternative devices for educational inclusion in common education schools". In this study we set out to know the socio-educational networks that are configured in the practices that these "supports" deploy in common education schools. From a socio-anthropological research approach, we develop field work in primary schools and carry out the construction of information from interviews, observations and field records.

The clipping of the study that we develop here allows us to know the continuities and discontinuities in which the tasks of the therapeutic companions are reconfigured, in a context of structural crisis of the educational system of Chubut and in which complex educational practices overlap in times pandemic COVID-19.
\end{abstract}

Keywords: Therapeutic companions - Education - Teacher's fight - COVID-19 pandemic - Inclusion.

\section{Introducción}

En este artículo nos proponemos avanzar en el análisis de las modalidades que asume el trabajo del acompañante terapéutico (AT) en escuelas públicas en contextos de "lucha docente" y de pandemia de COVID-19 en la ciudad de Comodoro Rivadavia, provincia de Chubut. Nuestro acercamiento a las escuelas en el trabajo de investigación que desarrollamos nos aproxima a conocer lo que entendemos como un contexto de crisis del sistema educativo en la jurisdicción. La crisis de la educación pública se expresa en la precarización laboral de los y las docentes, en el deterioro de las condiciones edilicias de las escuelas, en las partidas presupuestarias insuficientes y en la suspensión de las clases debido a los "paros" y a la "retención de servicios" de los/as trabajadores/as de la educación que se iniciaron en el año 2018 y se sostuvieron durante la segunda parte del ciclo lectivo 2019.

Debido a la medida de "Aislamiento Social Preventivo y Obligatorio" (ASPO)2, iniciada en marzo del año 2020, los Ministerios de Educación de la Nación y de la provincia (Resolución 2020-108-APN-ME y Resolución ME Chubut 38/2020), disponen la "continuidad pedagógica" mediada por "distintos soportes" (aulas virtuales, cuadernillos, entre otros) para mantener la escolarización. Consideramos que esta decisión pone en tensión la situación estructural del sistema educativo, en cuanto a las condiciones del trabajo docente y el cumplimiento de los derechos laborales, los recursos económicos para garantizar la conectividad tanto de estudiantes como de docentes, las desigualdades sociales y económicas que se inscriben en los distintos territorios, entre otros aspectos.

Estas condiciones atraviesan a distintos actores escolares que en los últimos tiempos, en contextos de políticas socio-educativas vinculadas a la "inclusión" educativa, se "incorporan" como formas alternativas de "apoyo" y de "acompañamiento" para estudiantes con discapacidad y/o estudiantes considerados/as con dificultades y problemas en sus aprendizajes. En la jurisdicción se trata de los/as AT, maestras de apoyo a la inclusión, auxiliares escolares para inclusión, a quienes las normativas educativas reconocen como "configuraciones de apoyo" a las trayectorias educativas de estudiantes en escuelas de educación común.

En esta perspectiva de análisis, nos interesa documentar las diversas modalidades que asumen el trabajo del AT, entendidas en términos de continuidades y discontinuidades en contextos de "lucha docente" y de pandemia de COVID-19. Lo hacemos en el marco del Proyecto de Investigación "Los 'apoyos' a alumnos/as. Dispositivos alternativos para la inclusión educativa en las escuelas de educación común" que venimos desarrollando en dos escuelas de educación común de la ciudad de Comodoro Rivadavia provincia de Chubut. En esta investigación nos proponemos conocer las tramas socioeducativas que se configuran en las prácticas que despliegan los "apoyos" en escuelas primarias.

\footnotetext{
${ }^{1}$ De aquí en adelante AT.

${ }^{2}$ De aquí en adelante ASPO.

${ }^{3}$ Proyecto de Investigación 'Los 'apoyos' a alumnos/as. Dispositivos alternativos para la inclusión educativa en las escuelas comunes". Directora Gladis Diaz. Res. De Rectorado 559/17.NPI 1369. 2017-2020.UNPSJB.
} 


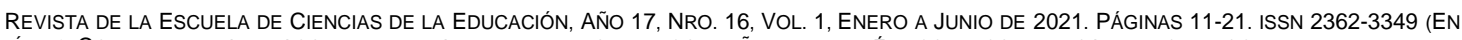
LÍNEA). CONTINUIDADES Y DISCONTINUIDADES EN EL TRABAJO DEL ACOMPAÑANTE TERAPÉUTICO EN CONTEXTOS DE LUCHA DOCENTE Y DE PANDEMIA DE COVID-19 EN ESCUELAS PÚBLICAS DE LA PROVINCIA DE CHUBUt. GLADIS NOEMÍ, DíAZ. CRISTINA DEL VALLE, PEREYRA. CYNTHIA SOLEDAD, NEUN.

Nos interesa señalar que el trabajo de investigación durante los años 2019 y 2020 está atravesado por las interrupciones de las clases presenciales y por las particularidades que asumen las prácticas escolares en estas circunstancias. En este tiempo mantuvimos entrevistas presenciales y telefónicas con algunas AT de las escuelas donde realizamos nuestro trabajo de campo. Y de este modo pudimos reconocer que la tarea AT en torno a niños y niñas con discapacidad que asisten a escuelas primarias, adquieren diversas modalidades respecto a los espacios laborales y las tareas, los vínculos con las docentes, las familias y los/as profesionales de salud y fundamentalmente en relación con los procesos de enseñar y de aprender. Estas modalidades de trabajo presentan continuidades y discontinuidades, en la reconfiguración que asumen en tiempos de "lucha docente" y de "pandemia", respecto de las prácticas que desarrollan en las escuelas en tiempos de presencialidad.

\section{Contexto de crisis socioeducativa en Chubut}

Desde el año 2018 el gobierno provincial despliega diversas medidas políticas que impactan en la situación estructural del sistema educativo de Chubut, las cuales se expresan en la falta de pago en tiempo y forma del salario docente y de otros/as trabajadores de la educación, recortes en las partidas presupuestarias (fundamentalmente para alimentos y productos de limpieza), deficiencias en el mantenimiento de los edificios escolares -que provocó que muchos de ellos no fueran habitables- e incumplimientos en el pago del Transporte Educativo Gratuito (TEG). En paralelo, una creciente mercantilización de la educación que se manifiesta en las "listas de espera" de las escuelas privadas en distintas ciudades de la provincia.

Estas situaciones llevaron a que en el año 2019 se profundicen medidas de fuerza a través de paros docentes, retención de servicio y otras modalidades como cortes de rutas, marchas y manifestaciones en espacios públicos. Durante ese ciclo lectivo la "lucha docente" superó los cien días sin clases y se sostuvo hasta mediados de noviembre, lo cual provocó que en muchas escuelas no se concrete la finalización del ciclo lectivo.

Durante el receso escolar de verano se continuó con el atraso y el pago "escalonado" ${ }^{4}$ del salario. En este contexto el inicio del ciclo lectivo 2020 estuvo atravesado por la continuidad de estos conflictos y por un fuerte reclamo por parte de los/las docentes donde se le solicitaba al gobierno provincial garantías respecto a: arreglos en los edificios escolares, actualización de las partidas presupuestarias para las escuelas, pago del salario en tiempo y forma, pago de las cláusulas gatillo, entre otros.

Finalmente, el no inicio de las clases se concreta en la mayoría de las escuelas públicas de las distintas localidades de la provincia de Chubut, debido al pago escalonado y un atraso -de dos meses- en el sueldo de los/las trabajadores/as de la educación.

Consideramos que asistimos, desde hace varios años, a una profundización de políticas neoliberales que se expresan en el desfinanciamiento de la educación pública y en la retirada del Estado como garante de la misma. Como consecuencia de un contexto estructural neoliberal, signado por el capitalismo, que como tendencia hegemónica produce transformaciones económicas, políticas, sociales y culturales desde una lógica economicista que genera procesos de fragmentación sociocultural en prácticas sociales y escolares (Achilli, 2010).

En este escenario, la pandemia de COVID-19 en Chubut agudiza la crisis estructural del sistema educativo y sus efectos en los procesos escolares de los/as estudiantes. Las medidas de ASPO dan continuidad a ciclos lectivos interrumpidos desde 2018 por la crisis del sistema educativo y las consecuencias mencionadas.

Por último, nos interesa señalar que esta crisis estructural se produce en tiempos en que la legislación educativa -Ley Nacional de Educación, 2006 y la Ley de Educación de Chubut, 2010- impulsa la inclusión educativa a través de normativas que intentan regular las prácticas escolares. Sin embargo, la retórica "inclusiva" colisiona con ciertas condiciones materiales y simbólicas -producto de la lógica neoliberal que penetra la vida cotidiana de las escuelas- que no posibilitan los cambios necesarios para evitar la exclusión.

\section{Acerca de nuestra investigación}

Los procesos socioeducativos inherentes a las transformaciones dirigidas a promover la "inclusión" educativa se producen en un entramado de discursos y prácticas en el que surge la "necesidad" de solicitar "apoyos" personales, entre ellos "Acompañantes Terapéuticos", para alumnos/as que transitan la educación común. Dicha "necesidad" se constituye a partir de la identificación por parte de los/as docentes de "problemas de aprendizaje" y/o de "conducta" en algunos/as alumnos/as, que presentan dificultades reales o supuestas para cumplir con lo esperado por las formas escolares y en aquellas situaciones en que los/as niños/as tienen diagnósticos o informes de profesionales de salud. Siendo muchas veces la presencia de estas figuras condición para continuar su trayecto educativo en la escuela.

Estas estrategias de "apoyo" se configuran en el marco de políticas públicas -tanto nacionales como provinciales- que intentan garantizar la "inclusión" educativa. Suponen cambios en las formas

${ }^{4}$ Modalidad de pago de los sueldos por rango a los empleados estatales-salud, educación y justicia- dependientes de la administración pública de la provincia de Chubut. Se inicia en el 2018 y continua vigente en el año 2020 


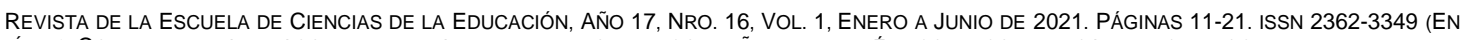
LÍNEA). CONTINUIDADES Y DISCONTINUIDADES EN EL TRABAJO DEL ACOMPAÑANTE TERAPÉUTICO EN CONTEXTOS DE LUCHA DOCENTE Y DE PANDEMIA DE COVID-19 EN ESCUELAS PÚBLICAS DE LA PROVINCIA DE CHUBUT. GLADIS NOEMÍ, DíAZ. CRISTINA DEL VALLE, PEREYRA. CYNTHIA SOLEDAD, NEUN.

escolares, en las relaciones entre los/as diferentes agentes educativos y en las prácticas institucionales, sin embargo no siempre logran revertir los procesos de exclusión.

A partir de esta problemática, en el Proyecto 'Los 'apoyos' a alumnos/as. Dispositivos alternativos para la inclusión educativa en las escuelas comunes" nos proponemos construir conocimiento acerca de las prácticas y sentidos que los/as actores educativos producen en torno a los denominados "apoyos" a alumnos/as del primer ciclo de escuela común, entre ellos los AT.

La investigación se funda en un marco epistémico relacional para comprender a los/as sujetos/as, las prácticas y los procesos escolares. Desde los enfoques socioculturales en psicología educacional concebimos al aprendizaje como situado, donde los/as sujetos/as y las situaciones se implican mutuamente (Baquero, 2014; Elichiry, 2010). Son las situaciones escolares las que promueven distintos modos de participación, de interacción y de apropiación y en este marco los "apoyos" se comprenden como sistemas de acompañamiento. Constituyen una "trama de sostén" construida por agentes escolares y no escolares que superan a aquellos "apoyos" desarticulados que existen en las escuelas (Maddoni, 2014). Son sistemas de apoyo a la enseñanza y al aprendizaje que organizan condiciones y mediaciones que posibilitan el aprendizaje escolar de los/as estudiantes, eludiendo algunos obstáculos propios del dispositivo escolar (Aizencang, et al., 2016).

Respecto de la orientación teórico-metodológica, el proceso de investigación se desarrolla desde un enfoque relacional-dialéctico (Achilli, 2005), que supone considerar los procesos bajo estudio en el cruce de interrelaciones dialécticas, como parte de una totalidad concreta (Kosik, 1967). Desde este enfoque entendemos a la problemática de nuestra investigación en la articulación de distintos niveles analíticos: los cotidianos sociales, en los que los actores educativos producen prácticas y sentidos en torno a los procesos denominados de "apoyos" a alumnos/as en escuelas de educación común, a las políticas socio-educativas, las que intentan regular y orientar las prácticas en torno a estos "apoyos" en procesos de inclusión educativa y los procesos históricos más generales, en términos de relaciones sociales históricas. Esta perspectiva teórico-metodológica nos permite re-construir las relaciones entre los procesos en estudio en el interjuego de la dinámica de esos niveles analíticos (Achilli, 2005).

El trabajo de campo se inicia en el año 2018 en el primer ciclo de dos escuelas públicas de nivel primario, en la ciudad de Comodoro Rivadavia. Estas escuelas se encuentran ubicadas en distintas zonas, una emplazada en el "centro" y la otra, en un barrio de la zona "norte", alejado del centro de la ciudad.

Este estudio es de carácter intensivo y por tanto se nutre de distintas estrategias de construcción de la información que posibilitan el acceso en profundidad a los sentidos y las prácticas que construyen los/as sujetos/as: observaciones, entrevistas, entrevistas grupales. Poner en juego estas estrategias teórico-metodológicas supone llevar adelante un trabajo de campo en el que vamos construyendo una relación intersubjetiva que "resulta, desde el punto de vista metodológico, crucial en el análisis interpretativo y en la contextualización del proceso de investigación" (Achilli, 2005, p. 64).

Las observaciones las llevamos a cabo en distintos espacios y momentos escolares: aulas, recreos, entradas y salidas, bibliotecas y en salones de educación física, música y educación artística. Las entrevistas las realizamos con AT, docentes de educación común, directoras y vice directoras y maestras de apoyo a la inclusión del primer ciclo de las dos escuelas públicas de nuestro estudio. También, incluimos a profesionales de salud del ámbito privado de las áreas de psicología, fonoaudiología, psicopedagogía, que asisten a los/as niños/as con los/as cuales trabajan las AT.

A la vez, realizamos una revisión y análisis de documentaciones estatales a nivel nacional y provincial, referidas a políticas socio-educativas denominadas de inclusión educativa, y de los textos elaborados por los/as sujetos/as de nuestra investigación: actas escolares, acuerdos institucionales entre AT y escuelas, informes y planificaciones docentes.

Respecto a las AT que participan en esta investigación, son todas mujeres y sus edades oscilan entre los 26 y 45 años. Algunas de ellas son estudiantes avanzadas de carreras universitarias como profesorado y licenciatura en ciencias de educación y de institutos de formación docente en educación física, primaria y educación especial. Otras, poseen título de acompañante terapéutico y/o estudian en universidades públicas o privadas la formación en acompañantes terapéuticos. La mayoría cuenta con experiencias previas de trabajo como AT en escuelas de educación común y en otros ámbitos como la salud mental. Sus condiciones laborales son diversas: contratadas por las obras sociales y/o por las familias de los/as niños/as.

Estas AT asisten de lunes a viernes a las escuelas junto a los/as niños/as con los/as cuales trabajan. Ingresan a las instituciones escolares en el mismo horario que el resto de los/as docentes y estudiantes, acompañan a los/as niños/as durante toda la jornada escolar y no suelen concurrir a las escuelas cuando ellos/as se ausentan.

Los y las niños/as con los/as cuales trabajan las AT tienen diagnósticos médicos vinculados a problemáticas definidas como subjetivas, emocionales y orgánicas, y cuentan con certificado de discapacidad. Todos/as ellos/as tienen obra social y acceden a distintas terapias.

El análisis desde nuestro enfoque implica un proceso "a través del cual se van generando distintas producciones tendientes a relacionar fragmentos de información empírica en sucesivos niveles de integración entre los mismos y con la necesaria intervención de las referencias teóricas" (Achilli, 2005, p. 86). Desde esta línea desarrollamos procesos analíticos de interpretación, contextualización, contrastación y reconstrucción (Rockwell, 2009). 


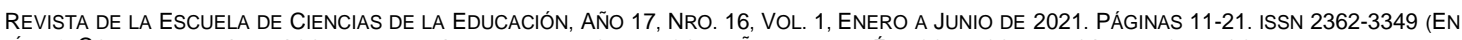
LÍNEA). CONTINUIDADES Y DISCONTINUIDADES EN EL TRABAJO DEL ACOMPAÑANTE TERAPÉUTICO EN CONTEXTOS DE LUCHA DOCENTE Y DE PANDEMIA DE COVID-19 EN ESCUELAS PÚBLICAS DE LA PROVINCIA DE CHUBUT. GLADIS NOEMÍ, DíAZ. CRISTINA DEL VALLE, PEREYRA. CYNTHIA SOLEDAD, NEUN.

En concordancia con este abordaje analítico, la lectura de los documentos internacionales y de las normativas nacionales y jurisdiccionales ${ }^{5}$ nos permite comprender las discontinuidades de sentido que asumen los "apoyos" en las escuelas, entre ellos del AT. Los documentos internacionales sustentados en concepciones del movimiento de la educación inclusiva (UNESCO, 2008) y las definiciones de la Convención Internacional de los Derechos de las Personas con Discapacidad (2006) coinciden en reconocer a los "apoyos personalizados/personales" para la inclusión educativa de "todos/as" y para "garantizar" el derecho a la educación de las personas con discapacidad. En este marco, la normativa nacional a través de las Resoluciones del CFE № 79/09 y № 155/11 refieren de modo escueto a "apoyos" "no docentes" o "privados" - sin hacer referencia al AT- que pueden constituir una "configuración práctica de atención" que "afecta la dimensión personal en un vínculo educativo para y con el estudiante con discapacidad". Estas normativas definen a las "configuraciones de apoyo" como redes, relaciones, posiciones, interacciones entre personas, grupos o instituciones que se conforman para identificar las barreras al aprendizaje y desarrollar estrategias educativas para la participación escolar (Res. CFE N79 (09) ${ }^{6}$. Sin embargo, podemos plantear que la normativa jurisdiccional define al AT como un "recurso de salud" (Circulares Técnicas 04/10, 02/14 y 01/16) y en las prácticas cotidianas como ese "apoyo" que las normativas nacionales reconocen.

También, en estos tiempos se amplía el acceso de las personas con discapacidad, a través del Certificado Único de Discapacidad (CUD), a prestaciones integrales de salud que contemplan al "acompañante" para garantizar la accesibilidad plena a todos sus derechos. Cabe aclarar que no se refiere al "acompañante terapéutico", que es una figura que surge en el campo de la salud mental, tal como lo reconoce la normativa educativa jurisdiccional.

Como decíamos, el trabajo de los/as AT en las escuelas se encuentra regulado por el Ministerio de Educación provincial a través de las Circulares Técnicas 04/10, 02/14 y 01/16 que establecen que el AT es "una figura que corresponde al área de salud" y que trabaja con "el paciente: niño/a, adolescente o adulto". Se reconocen como destinatarios/as de su intervención a "estudiantes con TGD (trastornos generalizados del desarrollo), autismo u otro trastorno del orden conductual y/o psiquiátrico que demanden una intervención terapéutica específica"7. Entre sus funciones se explicitan las siguientes: ser sostén frente a la ansiedad, miedos, angustias, facilitar la relación entre él/ella y su entorno afectivo, favorecer la participación activa y la integración del/la paciente a su medio social y ayudarlo/a a planificar, organizar y decidir cuando no esté en condiciones de hacerlo por sí mismo ${ }^{8}$.

Analizamos que estas normativas no consideran el carácter relacional de los "apoyos" definidos a nivel nacional, ya que no se hace referencia a la necesaria articulación de sus intervenciones con otros/as agentes escolares que toman decisiones en relación a las trayectorias escolares de los/las estudiantes. Tampoco refieren a las situaciones escolares, al omitir aspectos que den cuenta de la intervención del AT en un contexto particular como lo es la escuela. Estas regulaciones traducen la definición del AT como apoyo del campo de la salud, cuya prestación estaría garantizada con la presentación del CUD (art 10 de la Ley $24.901^{9}$ de acuerdo al art. 3 de la Ley $22.431^{10}$ ) a las obras sociales. A la vez, conciben al AT desde los sentidos originarios ligados a la psiquiatría que en la década de los 60 impulsó los movimientos de desmanicomialización en nuestro país (Rossi, 2012). El AT surge para acompañar, escuchar y contener a los pacientes psiquiátricos en sus tratamientos ambulatorios. Con el paso de los años, esta figura de la salud mental fue tomando cada vez mayor protagonismo y comenzó a trasladarse a otros espacios tales como la escuela, donde el AT es "un agente de salud que trabaja inserto en un equipo terapéutico, con una persona que está padeciendo una conflictiva de orden psíquico, emocional y que como consecuencia manifiesta síntomas en el plano educativo" (Rossi, 2012, p. 217).

Por otra parte, reconocemos que algunos autores plantean que la intervención del AT en las escuelas fue ampliándose, y que se solicita su intervención por estudiantes con discapacidad, trastornos graves en la infancia para la integración escolar (Rossi, 2012), por problemas de aprendizaje y de conductas no esperadas por la escuela (Viscaino, 2011). También, se plantea que el desajuste entre las políticas de inclusión educativa y las condiciones de las escuelas comunes para incluir a los/as estudiantes con discapacidad, ha incrementado la demanda de padres, profesionales y escuelas que solicitan el AT para que los/as niños/as reciban atención personalizada (Mont, 2016).

Más allá de las prescripciones de la normativa, consideramos que en las escuelas si bien se reconoce al AT como "apoyo" del campo de la salud, las expectativas son diversas respecto de su trabajo, por lo cual emergen tensiones que resuenan en sus interacciones con los/as agentes escolares y en las prácticas que asumen.

\footnotetext{
${ }^{5}$ Nos referimos a las normativas elaboradas por el Ministerio de Educación de la provincia de Chubut.

${ }^{6}$ La Resolución CFE № 79/09 establece como normativa el documento "Educación Especial. Una modalidad del Sistema Educativo en Argentina. Orientaciones 1" elaborado por López (2009). Ministerio de Educación de la Nación 1ª. Ed. Buenos Aires.

${ }^{7}$ Circulares Técnicas del Ministerio de Educación de la provincia de Chubut № 04/10, № 02/14 y № 01/16. 8 Ídem.

${ }^{9}$ Ley 24.901 Sistema de prestaciones básicas en habilitación y rehabilitación integral a favor de las personas con discapacidad. Honorable Congreso de la Nación Argentina. 1997.

${ }^{10}$ Ley 22.431 Sistema de protección integral de los discapacitados. Poder Ejecutivo Nacional. 1981.
} 


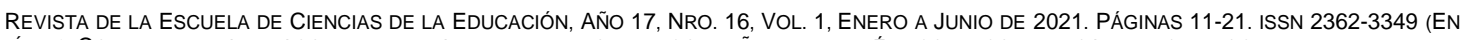
LÍNEA). CONTINUIDADES Y DISCONTINUIDADES EN EL TRABAJO DEL ACOMPAÑANTE TERAPÉUTICO EN CONTEXTOS DE LUCHA DOCENTE Y DE PANDEMIA DE COVID-19 EN ESCUELAS PÚBLICAS DE LA PROVINCIA DE CHUBUT. GLADIS NOEMÍ, DíAZ. CRISTINA DEL VALLE, PEREYRA. CYNTHIA SOLEDAD, NEUN.

En este sentido, hallamos que las tareas que realizan las AT son heterogéneas y que no sólo "asisten" a los/as niños/as ante una "crisis" o los/as "sostienen" para que estén "disponibles" ante las demandas del aprendizaje escolar. Muchas de sus intervenciones están comprometidas con la enseñanza, cuando realizan actividades vinculadas al diseño y al desarrollo de las "adecuaciones curriculares", desdibujándose su labor del orden de la salud. En algunas situaciones, a partir de sus intervenciones con los/as niños/as en las escuelas, también se vinculan con profesionales de salud de distintas áreaspsiquiatría, psicología, psicopedagogía, fonoaudiología y terapia ocupacional-que pertenecen a centros de atención privada.

\section{El trabajo de las "acompañantes terapéuticas"11 en tiempos en que los niños y las niñas no asisten a la escuela}

La pandemia de COVID-19 se inscribe en un contexto provincial de crisis de la educación pública en Chubut, por lo que sostenemos que dio continuidad a la "no presencia" de muchos/as niños, niñas y jóvenes en las escuelas iniciada ante las medidas de lucha docente que se desarrollan desde el año 2018.

La medida de ASPO y la "continuidad pedagógica" a través de la enseñanza mediada por la tecnología supuso tanto para docentes como para estudiantes, el desplazamiento del espacio público al privado, incorporando actividades escolares a los hogares. Así, la "organización de aulas virtuales", la "comunicación por las redes sociales", "los audios y mensajes de textos por WhatsApp", significaron distintas estrategias desarrolladas por los/as docentes con el objetivo de continuar con la escolarización de niños, niñas y jóvenes. También, se sumaron la "distribución de los cuadernillos impresos" elaborados por el Ministerio de Educación Nacional, la "difusión de contenidos educativos" desde la televisión pública, entre otros. Las entrevistas en profundidad realizadas con las AT en contextos de "lucha docente" y de "ASPO" nos permiten conocer y analizar las diversas maneras por las cuales intentan dar continuidad a su trabajo con los/las estudiantes, reconfigurando sus intervenciones cotidianas, a la vez que le otorgan diferentes sentidos.

\section{Modalidades de trabajo de las AT en relación con el espacio y las tareas}

Uno de los aspectos más recurrentes que las AT reconocen que se ha modificado en relación con el trabajo en contextos de "lucha docente" y de "pandemia" refiere al espacio laboral. La mayoría de ellas manifiestan que ante las interrupciones de clases en las escuelas dan continuidad a sus intervenciones en los hogares de los/as niños/as y/o en los domicilios de las AT:

Ante la situación escolar del año pasado de paros docentes, seguimos trabajando de manera continua en mi domicilio. Trabajábamos en el mismo horario escolar de cada uno de los nenes, de lunes a viernes (Registro de campo N²3-AT B, abril 2020).

Estas modificaciones en los lugares de trabajo implican para las AT "acondicionar" y "adaptar" los espacios domésticos en lugares para la enseñanza y el aprendizaje:

se trabaja en mi domicilio, el cual tuve que adecuar para poder generar el espacio de trabajo y que las jornadas sean armónicas y que los nenes estén cómodos (Registro de campo N²3-AT B, abril 2020).

Las AT realizan sus tareas acompañando al niño/a en la escuela, por lo cual la ausencia de clases presenciales, es considerada por las familias y las mismas AT una "disminución" en horas de su trabajo. Situación que, en algunos casos, les "impuso" desarrollar su labor en el hogar de los/as niños/as. Esta situación significa para las AT interactuar con una dinámica familiar "desconocida" y que puede resultar "difícil" cuando se intenta cumplir objetivos escolares, tal como lo expresan:

Actualmente voy dos veces a la semana al domicilio, aun así, es muy difícil trabajar porque no tiene una rutina y como no va a la escuela se levantan tarde, a veces llego y todavía está durmiendo, mientras se levanta y desayuna se nos pasa la hora (...) (Registro de Campo № 27AT P, mayo 2020).

La dinámica de la vida familiar constituye un contexto diferente al escolar, donde las regulaciones de tiempo, espacio, del trabajo y del modo de relacionarse difieren de las que imponen las formas escolares en sus rutinas institucionalizadas.

También, algunas AT manifiestan que tuvieron que dejar de acompañar a los/as niños/as. En algunas situaciones fueron por decisión de la familia "ante los casos de coronavirus en la ciudad, la familia me pidió que deje de ir por seguridad" (Registro de campo N²9 - AT C, junio 2020). En otras situaciones porque la demanda de las actividades escolares desconoce la singularidad de los niños/as en relación a este nuevo contexto del aprendizaje, tal como lo dice una AT "Esta semana suspendimos todo, porque él

\footnotetext{
${ }^{11}$ Nos referimos a "acompañantes terapéuticas" dado que todas las AT de nuestra investigación son mujeres.
} 


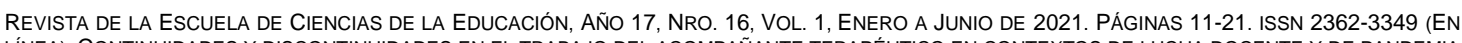
LÍNEA). CONTINUIDADES Y DISCONTINUIDADES EN EL TRABAJO DEL ACOMPAÑANTE TERAPÉUTICO EN CONTEXTOS DE LUCHA DOCENTE Y DE PANDEMIA DE COVID-19 EN ESCUELAS PÚBLICAS DE LA PROVINCIA DE CHUBUt. GLADIS NOEMÍ, DíAZ. CRISTINA DEL VALLE, PEREYRA. CYNTHIA SOLEDAD, NEUN.

no está respondiendo favorablemente a estas nuevas formas. Él también suspendió las terapias porque lo estresan demasiado" (Registro de campo № 22 AT C, mayo 2020). Más adelante, describiremos cómo la precarización del trabajo de las AT también es causa de que algunos/as niños/as se queden sin el "apoyo" que necesitan para el aprendizaje escolar.

En contextos de "pandemia" y de "aislamiento social y obligatorio", la mayoría de las AT trabajan con los niños y las niñas con discapacidad a través de "videollamadas", en las "plataformas virtuales" y/o "comunicaciones con las familias y las docentes":

Ahora estoy trabajando de manera virtual, realizando actividades y enviándolas por mail. Y acompañando desde mi lugar lo que van subiendo al Classroom (Registro de campo $N^{\circ} 23-\mathrm{AT}$ B, mayo 2020).

Estoy adaptando las actividades y se las entrego a los profesores y las videollamadas con los chicos ayudan a tener un orden (Registro de campo 22-AT C, mayo 2020).

En estas tareas las AT "resignan" el trabajo individual con el/la niño/a para ampliar sus intervenciones con docentes, familias o terapeutas. En particular, nos interesa mencionar que en tiempos de "lucha docente" y de ASPO se modifica la modalidad de trabajo de las AT, pero sus tareas continúan vinculadas con promover los aprendizajes de los/as niños/as. Son recurrentes los planteos en las AT en relación a que asumen tareas vinculadas a la enseñanza que en algunos casos implica la toma de decisiones en torno al "qué" y al "como" enseñar:

En relación con los paros docentes, se siguió trabajando, reforzando los contenidos que se alcanzaron a dar en la primera parte del año, mediante las actividades del libro de grado, modificando actividades, buscando recursos y material didáctico concreto para poder darle continuidad al ritmo que tenía en el aula (Registro de campo № 23-AT B, mayo 2020).

En la casa de T trabajamos con los cuadernillos de nación porque desde la escuela nunca le enviaron nada. De esos cuadernillos sacaba algunas actividades y las adecuaba para que él las entendiera (Registro de campo N²9 - AT C, junio 2020).

Analizamos dos cuestiones importantes respecto a las actividades pedagógicas asumidas por las AT. Por un lado, señalan que ellas "adaptan" las actividades que proponen los y las docentes; las cuales, en su mayoría, no considerarían las particularidades y necesidades de los niños y niñas": "no solo tengo que intervenir como docente, sino tengo que hacer adaptaciones curriculares, ya que los profesores no me mandan trabajos adaptados a él, así que los tengo que hacer yo" (Registro de campo №24-AT C, mayo 2020).

Y por otro, reconocemos que las AT en esta tarea de "adaptar" las actividades toman decisiones orientadas a ofrecer propuestas alternativas y accesibles en relación a las "particulares" maneras de aprender de los/as niños/as:

siempre le hice las adecuaciones, así que continué haciéndolas, armo videos explicativos para la nena, su nivel es muy bajo y no tiene buena atención, busco diferentes estrategias para que entienda las consignas de forma virtual (Registro de campo № 26-AT. G, mayo 2020).

Consideramos que los cambios en los espacios y la modalidad de intervención producen nuevas condiciones de trabajo. El asistir a los domicilios de los/as niños/as implica un traslado a lugares diferentes al realizado cuando se asiste a la escuela:

se me complicó bastante cuando empezó todo esto porque el nene vive en Bellavista Norte y yo en el Roca, la distancia es bastante grande y a eso se suman los horarios y controles de circulación (Registro de campo N²7-AT. P, junio 2020).

Asimismo, el acompañamiento de modo no presencial a los/as niños/as en relación con la propuesta de actividades escolares enfrenta al AT con limitaciones y tareas que se traducen en un trabajo mayor, difícil y que insume más tiempo:

trabajo desde mi casa. Me lleva mucho más tiempo trabajar de esta forma, no se termina nunca, no es como antes que llegaba a mi casa después de la escuela y listo. Para mí ahora es re difícil la situación porque me lleva el triple de tiempo y hay cosas que yo no puedo hacer sola porque soy acompañante y desde la escuela no me ayudan (Registro de campo №28-AT A, mayo 2020).

Entendemos, que el trabajo de las AT en las nuevas condiciones visibiliza la complejidad del mismo que se expone en las demandas actuales y las de siempre, y que se traduce en la emergencia de dificultades y limitaciones no siempre reconocidas. Por esto, consideramos que las tareas desplegadas por 


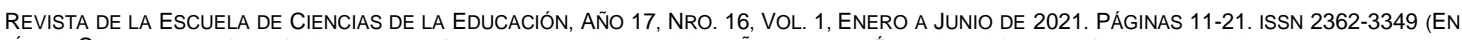
LÍNEA). CONTINUIDADES Y DISCONTINUIDADES EN EL TRABAJO DEL ACOMPAÑANTE TERAPÉUTICO EN CONTEXTOS DE LUCHA DOCENTE Y DE PANDEMIA DE COVID-19 EN ESCUELAS PÚBLICAS DE LA PROVINCIA DE CHUBUT. GLADIS NOEMÍ, DíAZ. CRISTINA DEL VALLE, PEREYRA. CYNTHIA SOLEDAD, NEUN.

las AT se han reconfigurado ante la no asistencia de los/as niños/as a las escuelas en la tensión entre las continuidades y los nuevos desafíos. Éstos tienen que ver con un nuevo espacio de trabajo como lo es el domicilio de los/as niños/as o de las AT, pregnado de las dinámicas familiares, y el desarrollo de actividades con nuevos soportes tecnológicos que intentan mediar las propuestas escolares. Las continuidades se relacionan con la "fluida" comunicación con las familias en relación con el modo de trabajo y con la asunción de actividades vinculadas con la enseñanza, ambas tareas que en los nuevos contextos adquieren mayor intensidad.

Estas continuidades y nuevos desafíos son significados por las AT como disruptivos para su trabajo que en la cotidianeidad escolar se define como de "acompañamiento" a un/una niño/a en un contexto específico para aprender como lo es la escuela. Las interrelaciones de las AT con otros/as actores escolares y no escolares nos permiten analizar las particularidades de su trabajo en relación a poder inscribirse en un sistema de acompañamiento como un "tejido particular que se va armando tanto al interior de la escuela como entre la escuela y otros contextos de aprendizaje, aportando saberes y herramientas que abren a otros rumbos" (Aizencang et al., 2015).

\section{Modalidades de trabajo en torno a las vinculaciones con otros/as actores/as escolares y no escolares}

En este eje de análisis nos interesa abordar las vinculaciones emergentes que se tejen entre las AT y otros/as agentes implicados/as en los procesos de enseñanza y de aprendizaje de las/os niñas/os en tiempos de "lucha docente" y de "pandemia". Entre estos/as actores/as identificamos docentes, familias y profesionales de la salud privada.

Las AT comparten con las docentes la cotidianidad del aula pero ante el "paro docente" y "retención de servicio" este encuentro y los intercambios se suspenden. Por otra parte, en tiempos de ASPO los intercambios de las AT con algunas de las docentes del aula se concretan, fundamentalmente, a través de diferentes de medios de comunicación tales como el correo electrónico, las plataformas virtuales, las videollamadas por WhatsApp, llamadas telefónicas, entre otras. Dichas interrelaciones se construyen en torno a los procesos de enseñar:

tenemos una comunicación con profesores y directivos a través de videollamadas para ponernos de acuerdo en cómo vamos a trabajar los contenidos...Las adaptaciones yo las hago, se las envío a los docentes, ellos la suben a la plataforma (Registro de campo №22-AT C, mayo 2020).

Sin embargo, en las voces de algunas AT resuena que la situación de pandemia "las deja trabajando en soledad", las rutinas escolares se modificaron drásticamente en el tiempo y en el espacio, así como las posibilidades de relacionarse con otros/as actores sociales comprometidos con los procesos de aprendizaje de los/as niños/as:

y ahora tengo que hacer todo yo sola, la maestra me manda un montón de actividades escaneadas y ni siquiera son para el nivel que tiene $B$, son re difíciles. Tengo que buscar actividades que más o menos sean para él, armarlas y enviarlas a su mamá por email (Registro de campo N²8 AT-A, mayo 2020).

Consideramos que las posibilidades y modos de vincularse entre las AT y las docentes del aula dan cuenta de dos cuestiones. Por un lado, el hecho de que muchos/as docentes se encuentran realizando "paro" y "retención de servicios" ante la falta de pago de sus salarios en contextos de ASPO. Y por otro lado, las dificultades que los/as docentes y las AT siempre tienen para construir un modo de trabajo colaborativo dadas las condiciones laborales y la ausencia de regulaciones institucionales que posibiliten tiempos y espacios compartidos más allá del aula.

Si consideramos que las intervenciones de las AT están comprometidas con aspectos de la enseñanza, emerge como paradójico las pocas posibilidades de interrelaciones entre ellas y los/as docentes que poseen la "autoridad pedagógica" y la formación para asumir la responsabilidad de los procesos de enseñanza y de aprendizaje escolares.

Respecto a las interrelaciones de las AT con los/as terapeutas de los/as niños/as en tiempos de pandemia, algunas de ellas expresan que los intercambios se realizan a través de diferentes medios de comunicación, e incluso reconocen los aportes de los/as especialistas como sustanciales al momento de decidir las actividades de enseñanza para los/as niños/as:

yo me apoyo mucho en la psicopedagoga. Nosotras nos juntamos, ella comparte la terapia por videollamadas, yo le comparto cómo la nena trabaja conmigo. Siempre que tengo una pregunta acudo a ella porque ya la conoce (Registro de campo N²2-AT C, mayo 2020).

Podríamos plantear que la no presencialidad de los/as niños/as en las escuelas se traduce -en algunas situaciones- en la ausencia de la materialización de una enseñanza orientada a sus "necesidades" de aprendizaje, situación que moviliza los intercambios de las AT con los y las/as profesionales de salud del ámbito privado. 
REVISTA de LA Escuela de CienCIAS de LA EduCACIÓN, AÑo 17, NRO. 16, VOL. 1, ENERO A JUNIO de 2021. PÁGINAS 11-21. ISSN 2362-3349 (EN LÍNEA). CONTINUIDADES Y DISCONTINUIDADES EN EL TRABAJO DEL ACOMPAÑANTE TERAPÉUTICO EN CONTEXTOS DE LUCHA DOCENTE Y DE PANDEMIA DE COVID-19 EN ESCUELAS PÚBLICAS DE LA PROVINCIA DE CHUBUT. GLADIS NOEMÍ, DíAZ. CRISTINA DEL VALLE, PEREYRA. CYNTHIA SOLEDAD, NEUN.

En cuanto a las interacciones y las comunicaciones entre las AT y las familias, todas las entrevistadas acuerdan que las mismas son "fluidas" para sostener los procesos escolares de los/as niños/as. Para ello, las AT envían las actividades "adecuadas" a los/as niños/as y orientan a las familias en relación al desarrollo de las mismas:

enviamos actividades a la mamá para que las pueda hacer en la casa, si alguna se le complica hacemos video llamadas para explicarle como se hace. Algunas veces envían las actividades de la escuela, la mamá de B me avisa y me las envía (Registro de campo №27-AT P, junio 2020).

Así emerge una nueva tarea de las AT en tiempos en los que la escuela debe delegar a las familias la enseñanza de contenidos escolares. Se trata de acompañar a las familias en la mediación de la oferta de las actividades de enseñanza y de aprendizaje y también orientar en cuestiones tecnológicas que demandan las propuestas escolares virtuales.

En algunas ocasiones las comunicaciones entre las AT y las familias se fundan en su preocupación por los procesos formativos de los/as niños/as ante las actividades que se les proponen:

hablamos con la mamá para saber cómo está B. Me cuenta cómo va con las actividades. Tampoco sé si las está haciendo porque siempre me dice que está re bien y que hace todo, pero desde la escuela me informan que no está cumpliendo y no participa de las clases por zoom (Registro de campo №28-AT A, mayo 2020).

De este modo, las AT manifiestan que asumen la responsabilidad por el desarrollo de las actividades escolares en el hogar por parte de los/as niños/as, en tanto representaría su "inclusión" educativa, como lo es cuando pueden participar de las actividades en el aula gracias a su "acompañamiento".

Por otra parte, las AT que trabajan en los hogares manifiestan que las familias "colaboran" de diferentes maneras para que ellas puedan realizar con el/la niño/a las tareas escolares en un contexto con condiciones y una dinámica propia:

trabajo en su casa, la familia siempre colabora para que estemos tranquilos trabajando porque T se distrae fácilmente, igual es difícil trabajar porque su casa es muy chica y viven cinco personas (Registro de campo N²9-AT C, junio 2020).

Cabe destacar que las interrelaciones entre las AT y las familias están signadas por la condición laboral de las primeras que son contratadas/empleadas por estas familias, aun cuando "debieran" ser las obras sociales las encargadas de sus honorarios. Tal como una AT lo expresa: "los padres piden que vaya de manera presencial porque no estoy trabajando las horas que corresponden al presupuesto dado" (Registro de campo N²4-AT C, mayo 2020). Además, son las familias las que "controlan" el cumplimiento de su trabajo para su pago, a través de la asistencia:

no me dejaron de pagar nunca porque los papás me siguieron firmando la planilla de asistencia, aunque no pudiera trabajar de manera presencial con el nene, pero ellos entienden que no es mi culpa. Por el momento, solo voy dos veces y ellos me firman como si fuera todos los días (Registro de campo №27-AT. P, junio 2020).

Asimismo, entendemos que es la relación laboral que sostienen las AT con las familias, en condiciones de precariedad que ofrecen las obras sociales, la que define la asunción de responsabilidades respecto al acompañamiento a las familias. Responsabilidades que exceden las tareas de las AT y que no son significadas de este modo por los/as implicados/as en dicha relación laboral.

\section{Las condiciones laborales de las AT en tiempos de nuevas demandas}

Consideramos importante señalar que, en el marco de la pandemia, al mismo tiempo que se reconfiguran las tareas de las AT, sus condiciones laborales se ven atravesadas por diversas circunstancias de inestabilidad y precarización. Por un lado, las AT que realizan su trabajo a través de la "virtualidad" con los/as niños/as plantean que algunas obras sociales "reducen" los pagos dado la situación de "no presencialidad":

bajó lo que se me paga mensualmente, pagan menos porque no estamos cumpliendo la cantidad de horas reales. Los pagos son muy atrasados porque debemos presentar declaraciones juradas y demás papeles, que antes no lo requerían (Registro de campo №22-AT C, mayo 2020).

Por otro lado, las AT que asisten a los domicilios de los/as niños/as a realizar su trabajo expresan que las obras sociales solicitan diversos requerimientos y existen demoras en los plazos de aceptación/autorización para su labor y en los pagos de sus salarios: 


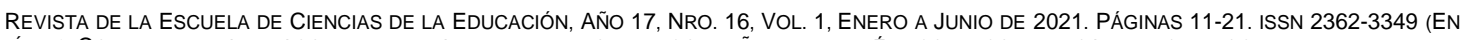
LÍNEA). CONTINUIDADES Y DISCONTINUIDADES EN EL TRABAJO DEL ACOMPAÑANTE TERAPÉUTICO EN CONTEXTOS DE LUCHA DOCENTE Y DE PANDEMIA DE COVID-19 EN ESCUELAS PÚBLICAS DE LA PROVINCIA DE CHUBUT. GLADIS NOEMÍ, DíAZ. CRISTINA DEL VALLE, PEREYRA. CYNTHIA SOLEDAD, NEUN.

...lo que te traba ahora no solo es la pandemia sino también cómo se están manejando las obras sociales, porque para trabajar en el domicilio solicitan el pedido del psiquíatra de acompañamiento domiciliario, para que la obra social autorice la prestación del AT (Registro de campo N²5-AT J, junio 2020).

Por otra parte, algunas AT manifiestan que dada la crisis económica y social, producto de la pandemia, muchas de las familias de los/as niños/as con quienes ellas intervienen han tenido dificultades en su situación laboral:

este mes, el papá de la nena se quedó sin trabajo y no puede seguir pagando la obra social y se dio de baja. Acordamos que no continuaba hasta que no le den el alta...Todo esto se da por lo que sucede actualmente, el papá no puede salir a trabajar, por ende, no tiene ingresos y eso afecta directamente mi trabajo (Registro de campo №22-AT C, mayo 2020).

Por último, concebimos que la situación de inestabilidad y precarización laboral de las AT que producen las obras sociales, no sólo vulnera los derechos laborales de las AT sino también los derechos a la salud y a la educación de los/as niños/as con discapacidad en situaciones que ven interrumpido su acompañamiento terapéutico. De este modo, la medicina enclavada en una economía de mercado laboral, se hace parte de una industria de la salud y de la enfermedad basada en el lucro y no en el derecho a la salud (Menéndez, 1987).

\section{Algunas conclusiones provisorias}

Un nuevo modo de hacer escuela, inesperado y no previsto, en tiempos de pandemia se inscribe en la crisis estructural de la educación pública de la provincia de Chubut que viene produciendo discontinuidades en el trabajo de los y las docentes, de las AT y en las trayectorias de aprendizajes de los/as niños/as.

Las AT en contexto de "lucha docente" y de "ASPO" intentan continuar con su trabajo a través de diversas modalidades de intervención, las cuales implican reconfiguraciones vinculadas a: los espacios laborales y las tareas, los vínculos con los/as docentes, las familias y los/as profesionales de salud, siempre comprometidas con la promoción de procesos de enseñanza y de aprendizaje. Estas modalidades de trabajo presentan continuidades y discontinuidades con las tareas que realizan en tiempos en que los/as niños/as asisten a las escuelas.

A pesar de la interrupción del trabajo en las escuelas, las AT dan continuidad a sus tareas en sus domicilios o en los hogares de los/as niños/as, lo cual implica "acondicionar" los espacios domésticos en lugares para la enseñanza y el aprendizaje escolar. Otras AT trabajan con los/as niños/as con discapacidad a través de "videollamadas", en las "plataformas virtuales" y se comunican con las familias y las docentes, "abandonando" la atención personal a los niños y las niñas. De este modo, se amplían e intensifican las tareas vinculadas a la comunicación virtual con los/as docentes, con las familias y en algunos casos con los/as profesionales de salud privada a través de medios tecnológicos. Comunicación en la que emergen nuevos modos de vincularse y de re-conocerse para el acompañamiento al aprendizaje escolar.

La continuidad de la intensificación de las interrelaciones de las AT con docentes, terapeutas y familias, y su puesta en valor, prefigura la trama de sostén que necesitan los procesos de aprendizaje de los/as niños/as con discapacidad en la escuela común. Sería esperable que en los tiempos por venir cambien las condiciones de trabajo de maestras/os y de AT, y los modos de organización escolar para viabilizar dicha trama.

Las nuevas tareas, disímiles a las que las AT desarrollan cotidianamente en las escuelas, se imbrican con aquellas vinculadas a la enseñanza que, en algunos casos, implican la toma de decisiones en torno al enseñar de modo ajustado a las necesidades educativas singulares de aprender de los/as estudiantes en situaciones heterogéneas y novedosas. De esta manera, son las AT, con los recursos simbólicos y materiales que disponen, las que están atentas a las dificultades que se añaden para participar y aprender bajo las formas escolares que subsisten más allá de la no presencialidad en las escuelas.

Las tareas que asumen las AT comprometidas con la enseñanza exponen una situación que excede a las intervenciones prescriptas por las normativas y a su formación específica. Entendemos que se pone en riesgo una propuesta formativa fundada en la justicia curricular (Connel, 2006) aun cuando se pretende dar continuidad a los aprendizajes de estudiantes más vulnerables.

Consideramos que los cambios en los espacios y la modalidad de intervención producen incremento de tareas y nuevas condiciones laborales que configuran un trabajo más oneroso, más difícil, que insume mayor tiempo y que las AT desarrollan en condiciones de precarización laboral. En estas circunstancias encarnan la contradicción de sostener lo escolar en la vida de los niños/as con discapacidad en tiempos de aislamiento social siendo un agente de salud privado.

Cuando el Estado se retira como garante del derecho a la educación pública las trayectorias formativas de los/as estudiantes se alteran, se vuelven discontinuas. En el caso de los/as niños/as con discapacidad los avatares son mayores aun cuando en nombre la "inclusión" educativa tengan un "apoyo". 
REVISTA de LA Escuela de CienCIAS de LA EduCACIÓN, AÑo 17, NRO. 16, VOL. 1, ENERO A JUNIO de 2021. PÁGINAS 11-21. ISSN 2362-3349 (EN LÍNEA). CONTINUIDADES Y DISCONTINUIDADES EN EL TRABAJO DEL ACOMPAÑANTE TERAPÉUTICO EN CONTEXTOS DE LUCHA DOCENTE Y DE PANDEMIA DE COVID-19 EN ESCUELAS PÚBLICAS DE LA PROVINCIA DE CHUBUT. GLADIS NOEMÍ, DíAZ. CRISTINA DEL VALLE, PEREYRA. CYNTHIA SOLEDAD, NEUN.

La presencia del mismo daría cuenta de que las situaciones escolares no pueden garantizar el aprendizaje escolar (Aizencang et al., 2015).

Nos preguntamos entonces si las practicas que las AT construyen no refieren a formas naturalizadas de entender la "inclusión" educativa, de modo tal que la incorporación del AT ya supondría una "práctica inclusiva" por parte de la escuela. Sobre todo cuando las AT, como ya mencionamos, se autoperciban como una "figura" que en sí misma "permite" desarrollar los procesos de "inclusión" escolar de niños/as con discapacidad.

Por último, proponemos reflexionar acerca de la tensión entre la relevancia de las prácticas de las AT como "sostén" del espacio escolar y los riesgos que suponen ciertas prácticas referidas a los apoyos a la "inclusión" educativa que producen acceso diferenciado a los aprendizajes escolares (Coicaud et al., 2008). Tensión en la que se produce la trayectoria escolar y la subjetividad de los/as niños/as con discapacidad.

\section{Referencias bibliográficas:}

Achilli, E. (2005). Investigar en Antropología Social. Rosario, Argentina: Laborde Editor.

Achilli, E. (2010). Contextos, hegemonía y cotidianeidad escolar. En: Escuela, familia y desigualdad social. Una antropología en tiempos neoliberales, pp.123-141. Rosario, Argentina: Laborde, Editor.

Aizencang, N., et. al. (2015). Pensar el aprendizaje en tanto situado: ¿Qué se entiende por acompañamientos al aprendizaje escolar? En: Memorias. VII Congreso Internacional de Investigación y Práctica Profesional en Psicología- XXII Jornadas de Investigación- XI Encuentro de Investigadores en Psicología del MERCOSURBuenos Aires. Psicología Educacional y Orientación Vocacional. TOMO 1, pp. 26-30. Ediciones Facultad de Psicología Universidad de Buenos Aires.

Aizencang, N.; Bendersky, B. y Maddonni, P. (2016). ¿Qué nos permite pensar el aprendizaje en tanto situado? aportes para el análisis de experiencias educativas. En: VIII Congreso Internacional de Investigación y Práctica Profesional en Psicología XXIII Jornadas de Investigación XII Encuentro de Investigadores en Psicología del MERCOSUR. Facultad de Psicología - Universidad de Buenos Aires, Buenos Aires.

Coicaud, C.; Diaz, G. y Pereyra, C. (2018). Relaciones entre la nominación de los "problemas de aprendizaje" y las solicitudes de "apoyo" de educación especial. En: Revista RUEDES. Red Universitaria de Educación Especial. N8, pp. 5- 29.

Connell, R. (2006). Escuela y justicia social (tercera edición). Madrid, España: Morata.

Kosik, K. (1967). Dialéctica de lo concreto. México: Editorial Grijalbo.

López, D. (2009). Educación Especial. Una modalidad del Sistema Educativo en Argentina. Orientaciones 1. Ministerio de Educación de la Nación 1a. Ed. Buenos Aires.

Maddoni, P. (2014). El estigma del fracaso escolar: nuevos formatos para la inclusión y la democratización de la educación. Ciudad de Buenos Aires: Paidós.

Mont, M. (2016). El acompañante terapéutico en la escuela. En: Revista Novedades Educativas № 300-301. Buenos Aires, Argentina

Menéndez, E. (1987). Modelo médico hegemónico. Modelo alternativo subordinado. Modelo de autoatención. En: Primeras Jornadas de Atención Primaria de la salud. Buenos Aires: Fundación Banco de la provincia de Buenos Aires.

Rockwell, E. (2009). La Experiencia Etnográfica. Historia y cultura en los procesos educativos. Buenos Aires, Argentina: Paidós.

Rossi, C. (2012). Acompañamiento terapéutico en el Ámbito Educativo. En: Dragotto, P.A. y Frank, M.L. (comps). Acompañantes. Conceptualizaciones y experiencias en A.T., pp. 205-21, Argentina: Brujas.

Viscaino, A. (2011). Las dificultades escolares en la actualidad. Sentidos del encuentro educativo. En: Actualidad Psicológica, N.․ 396, pp. 23-26.

Documentos:

Circular Técnica № 07. (2009). Dirección General de Educación Inclusiva. Ministerio de Educación de la provincia de Chubut, Argentina.

Circular Técnica No04. (2010). Dirección General de Educación Inclusiva. Ministerio de Educación de la provincia de Chubut, Argentina.

Circular Técnica Nº2. (2014). Dirección General de Educación Inclusiva. Ministerio de Educación de la provincia de Chubut, Argentina.

Circular Técnica N01. (2016). Dirección General de Educación Inclusiva. Ministerio de Educación de la provincia de Chubut, Argentina.

Ley de Sistema de Protección Integral de las personas con discapacidad №22.431. (1981). Sistema de Protección General al Discapacitado. Poder Ejecutivo Nacional. Buenos Aires, Argentina.

Ley de Sistema de Prestaciones Básicas en Habilitación y Rehabilitación Integral a Favor de las Personas con Discapacidad №24.901 (1997). Sistema de prestaciones básicas en habilitación y rehabilitación integral a favor de las personas con discapacidad. Honorable Congreso de la Nación. Ministerio Nacional de Justicia y Derechos Humanos. Buenos, Aires, Argentina.

Ley de Educación Nacional `26.206 (2006). Honorable Congreso de la Nación. Boletín oficial, 6 de febrero de 2007.

Ley de Educación Provincial VIII №91 (2010). Honorable Legislatura de Chubut. Boletín oficial Año LIII №11110,19 de noviembre del 2010. Chubut, Argentina.

Resolución № 79. (2009). Consejo Federal de Educación. Buenos Aires, Argentina.

Resolución №155. (2011). Consejo Federal de Educación. Buenos Aires, Argentina. 